\title{
Robot-Assisted Diagnostic Ultrasound - Design and Feasibility Experiments
}

\author{
S.E. Salcudean, G. Bell, S. Bachmann, W.H. Zhu, \\ P. Abolmaesumi, and P.D. Lawrence \\ University of British Columbia \\ Department of Electrical and Computer Engineering \\ Vancouver, B.C., Canada, V6T $1 Z 4$ \\ tims@ece.ubc.ca \\ http://www.ece.ubc.ca/ tims
}

\begin{abstract}
Motivated by the need for providing a better user interface for ultrasound technicians, a teleoperation approach to diagnostic ultrasound examinations is proposed in this paper. In this approach, the ultrasound probe is positioned by a robot, with the operator, the robot controller, and an ultrasound image processor having shared control over its motion.

An inherently safe, light, backdrivable, counterbalanced robot has been designed for carotid artery examinations. Its design, as well as experiments demonstrating effective free-motion and force control, are presented. The feasibility of using visual servoing for motion in the plane of the ultrasound probe has also been addressed. Using a modified image correlation algorithm, tracking of the carotid artery for periods of time in excess of ten seconds has been demonstrated.
\end{abstract}

\section{Introduction}

Medical ultrasound exams often require that ultrasound technicians hold the transducers in awkward positions for prolonged periods of time, sometimes exerting large forces. Not surprisingly, a number of studies indicate that they suffer from an unusually high incidence of musculoskeletal disorders (e.g. [1]).

Motivated initially by the need to alleviate these problems and present a more ergonomic interface to the ultrasound technician, the authors have embarked upon the development of a teleoperation system for medical ultrasound. The system consists of a master hand controller, a slave manipulator carrying the ultrasound probe, and a computer control system that allows the operator to remotely position the ultrasound transducer relative to the patient's body. The problem considered first as a test-bed for robot-assisted ultrasound was that of carotid artery examination. This examination is carried out to detect occlusive disease in the left and right common carotid arteries - a major cause of strokes.

A robot-assisted ultrasound examination system would provide other, not only ergonomic, benefits. For instance, since the location of the ultrasound transducer can be determined via the forward kinematics of the slave manipulator, 
three-dimensional ultrasound images can be reconstructed from a series of twodimensional image slices [4]. Remote probe positioning could also be used in teleradiology. Although a number of methods for transmitting ultrasound images have been proposed in the literature [3], none allow the radiologist to view and manipulate the ultrasound transducer at the remote site. The ability to position the ultrasound transducer in response to acquired ultrasound images would also be of benefit to image guided interventions (e.g., percutaneous pericardial puncture) and registration with past examination records or images obtained with other imaging methods (e.g., MRI).

This paper presents significant steps towards a robot-assisted ultrasound examination system. Based on ultrasound transducer position and force measurements taken during carotid artery examinations, a novel fully counterbalanced robot suitable for placing the ultrasound transducer in contact with a patient was designed and is presented in Section 2. The motion of the robot arm and the hand controller of the proposed ultrasound are based on measured positions and forces, acquired ultrasound images, and/or taught position and force trajectories. Several modes of control are discussed in Section 3, including the control of the transducer using ultrasound image tracking, for which the image Jacobian is derived. The feasibility of feature tracking in ultrasound images is discussed in Section 4, where it is shown that a modified correlation method can be used to track the carotid artery over periods of time as long as ten seconds. This will enable users of the system to apply image servoing techniques such as those described in [2], and can be used to follow along an anatomical feature such as a carotid artery, or to keep a feature, such as the tip of a needle, within the image plane.

\section{Ultrasound Robot Design}

\subsection{Measured Motion/Force Requirements}

The range of ultrasound probe motions and forces was measured during carotid artery examinations. These involve duplex imaging of the distal end of the common carotid artery and the proximal ends of the internal and external carotid arteries, both longitudinally and transversely, from the clavicle to the mandible on both the left and right sides of the neck.

A $5 \mathrm{MHz}$ ultrasound transducer used in the examination was fitted with a $\mathrm{JR}^{3}$ force/torque sensor and the magnetic field sensor of an ATC The Bird ${ }^{T M}$ electromagnetic position and orientation sensor,

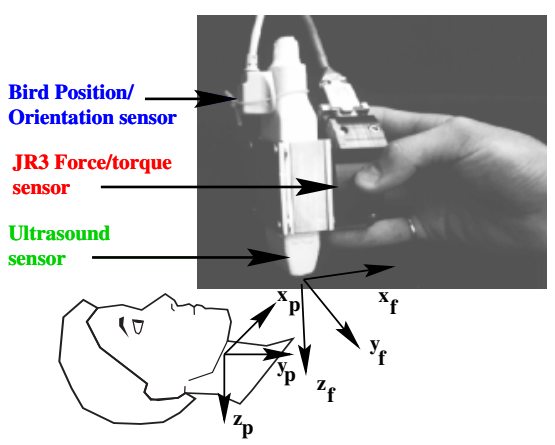

Fig. 1. Apparatus for force-torquepose experiment as shown in Fig. 1. The Bird's magnetic field generator was placed near the patient's neck. The ultrasound technician then carried out a carotid artery exami- 
nation on the patient, holding the force/torque sensor instead of the ultrasound transducer (Fig. 1).

Metallic objects in the proximity of magnetic sensors are known to cause significant measurement errors. These were quantified for our experimental setup by placing the instrumented ultrasound transducers in a number of known locations and reading the sensor measurements. An average error of less than $1 \%$ was found in orientation, and less than $30 \%$ for translation. The measured motion range and the maximum forces encountered are tabulated in Tables 1 and 2 . The uncertainty in translational motion range is not of significant concern, as the translational workspace of the robot must be significantly larger than measured in order to comfortably place the ultrasound probe against the patient. The orientation range can be approximated by the right elliptical cone

$$
\left(\tan 15^{\circ}\right)^{2} x_{p}^{2}+\left(\tan 35^{\circ}\right) y_{p}^{2}=z_{p}^{2}, \quad z_{p} \leq 0,
$$

shown, with the measured orientation data, in Fig. 2. Note that the transducer must be able to rotate by $\pm 45^{\circ}$ about its longitudinal axis everywhere within this right elliptical cone in order to scan transversely and longitudinally.

During these experiments, the maximum velocities recorded, of the order of $0.2 \mathrm{~m} / \mathrm{s}$

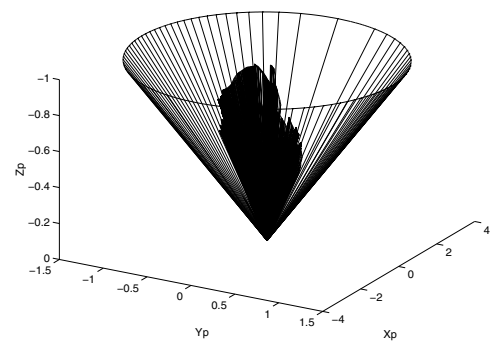

Fig. 2. Orientation range and $260^{\circ} / \mathrm{s}$, occurred during a switch to a different scanning area or during probe re-orientation. The average velocities were very small, of the order of $5 \mathrm{~mm} / \mathrm{s}$ and $3^{\circ} / \mathrm{s}$.

\begin{tabular}{|r|c||r|c|}
\hline \multicolumn{2}{|c|}{ Translation Range } & \multicolumn{2}{|c|}{ Angular Range } \\
\hline Axis & Translation & Axis & Rotation \\
\hline$x_{p}$ & $130 \mathrm{~mm}$ & $x_{p}$ & $35,-50 \mathrm{deg}$ \\
$y_{p}$ & $150 \mathrm{~mm}$ & $y_{p}$ & $-75,75 \mathrm{deg}$ \\
$z_{p}$ & $100 \mathrm{~mm}$ & $z_{f}$ & $-45,+45$ \\
\hline
\end{tabular}

Table 1. Probe Motion Estimate

\begin{tabular}{|c|c||c|c|}
\hline \multicolumn{2}{|c|}{ Force Range } & \multicolumn{2}{|c|}{ Torque Range } \\
\hline Axis & Force & Axis & Torque \\
\hline$x_{f}$ & $3.8 \mathrm{~N}$ & $x_{f}$ & $0.4 \mathrm{Nm}$ \\
$y_{f}$ & $4.2 \mathrm{~N}$ & $y_{f}$ & $0.7 \mathrm{Nm}$ \\
$z_{f}$ & $6.4 \mathrm{~N}$ & $z_{f}$ & $0.1 \mathrm{Nm}$ \\
\hline
\end{tabular}

Table 2. Probe Force Estimate

\subsection{Robot Design}

A robot used for ultrasound probe positioning must be safe under any circumstance, including power failure, and should move fast enough to allow the ultrasound examination to take place at a pace close to that achieved by the unassisted sonographer. Therefore, the robot should be light and of limited force ability to allow relatively rapid probe motion in a safe manner. The robot joints should be backdriveable so that the arm could be pushed out of the way if necessary and controlled effectively in force mode. In addition, the robot should cover the required range of motions and forces described above. Because of the large orientation workspace required, the orientation and transla- 


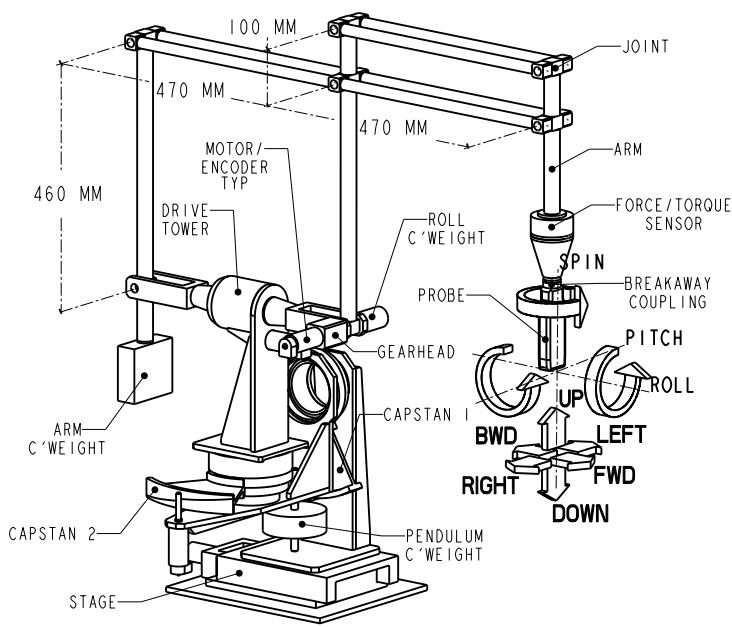

(a) Robot model

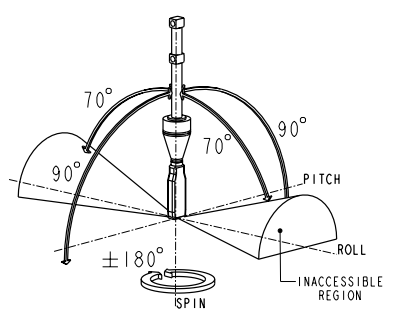

(b) Probe orientation workspace

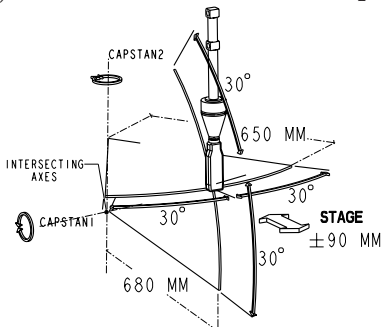

(c) Probe tip position workspace

Fig. 3. Ultrasound robot design and workspace

tion of the probe tip should be approximately decoupled. Otherwise, the entire robot arm has to be moved in order to accommodate probe orientation changes. This implies that the arm force capability has to be increased beyond a safe level, or that the arm has to be slowed down beyond an acceptable performance level. A number of design options, such as the use of a spherical wrist, were eliminated by testing whether the range of orientations in the elliptical cone (1) could be attained without interference and with reasonable link lengths. The only remote-center wrist structure that was found to satisfy the orientation range requirement was a rotating parallelogram linkage as used in the LARS robot [5]. The parallelogram linkage used in our design is different because of the need to counterbalance the arm.

The solid model of the robot is shown in Fig. 3, and a photograph is shown in Fig. 4. The robot is placed at the head of the bed, with the labeled LEFT, FWD and DOWN directions coinciding with the $x_{p}, y_{p}$, and $z_{p}$ axes of Fig. 1, respectively.

The probe is mounted on a breakaway magnetic coupling and its spin motor is housed in a

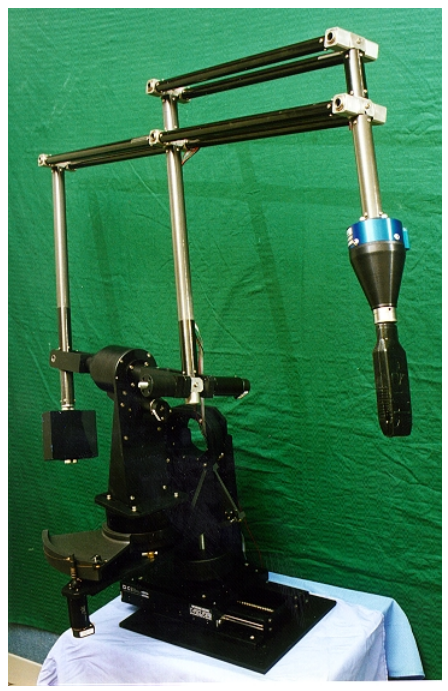

Fig. 4. Photograph of the ultrasound robot cone that attaches to a $\mathrm{JR}^{3}$ force/torque sensor supported by a light and stiff par- 
allelogram linkage. Constructed with carbon-fiber tubes and magnesium joints, this linkage weighs less than $2.4 \mathrm{~kg}$ including the ultrasound probe and its spin motor. A rigid drive tower with an offset motor rolls the parallelogram linkage. Two revolute intersecting axes actuated by capstan drives move the probe updown and left-right, and are mounted on a low friction $(<0.007 \mathrm{~N}-\mathrm{m})$ linear stage that moves the probe along the neck of the patient. Each capstan is mounted on a single crossed-roller bearing, and each uses $1 \mathrm{~mm}$ diameter coated, annealed, steel cable, pre-loaded with a tensioning device.

Revolute axes were chosen over translational stages in order to maximize the workspace and minimize the inertia seen at the probe. Note that the parallelogram wrist is rolled to one side or the other during most of the carotid artery examination, and therefore most of the probe nor-

\begin{tabular}{|l|l|l|c|}
\hline Axis & $\begin{array}{l}\text { Trans. } \\
\text { ratio }\end{array}$ & Motor & $\begin{array}{c}\text { Force/Torque } \\
\text { @ Current }\end{array}$ \\
\hline Roll & $93.6: 1$ & Maxon 90 & $12.28 \mathrm{~N} \mathrm{~m} \mathrm{@} \mathrm{2.5A}$ \\
Pitch & $50: 1$ & Maxon 90 & $6.56 \mathrm{~N} \mathrm{~m} \mathrm{@} \mathrm{2.5A}$ \\
Capstans & $40: 1$ & Maxon 90 & $10.50 \mathrm{~N} \mathrm{~m} \mathrm{@} \mathrm{5A}$ \\
Spin & $1: 1$ & Maxon 20 & $0.18 \mathrm{~N} \mathrm{~m} \mathrm{@} \mathrm{5A}$ \\
Stage & $0.49 \frac{\mathrm{rad}}{\mathrm{mm}}$ & Maxon 90 & $15 \mathrm{~N}^{*}$ @ 2.5A \\
\hline Probe Force & \multicolumn{3}{|c|}{$10-15 \mathrm{~N}^{* *}$} \\
\hline
\end{tabular}

Table 3. Actuation system characteristics mal force is controlled by capstan 2, which moves the least mass and therefore allows for higher bandwidth force-control.

Three adjustable counterweights were used to fully balance the robot. The roll and arm counterweights place the center of mass of the parallelogram linkage at a fixed point on the linkage roll axis, independently of the ultrasound transducer orientation. Because of mechanical interference (e.g. between the arm counterweight and the capstan), the roll axis had to be placed above capstan 1, creating an unstable inverted pendulum with the center of mass above the capstan 1 axis. This center of mass of this pendulum was moved below the capstan 1 axis by a pendulum counterweight.

As seen in Fig. 3 (b),(c), the ultrasound transducer workspace significantly exceeds the specified requirements.

\section{Teleoperation and Shared Control}

A signal flow diagram and the present hardware implementation for the teleoperation system are illustrated in Fig. 5.

During the ultrasound examination, the operator controls the ultrasound machine as usual, but moves a hand-controller or joystick instead of the ultrasound probe. Some of control modes that have been tested or are envisaged are: Master-slave mode without force feedback, with the ultrasound transducer velocity and force tracking a joystick displacement as described in the next subsection. A SpaceMouse/Logitech Magellan [6] was used as a joystick. Experimental results are described in Section 4.

Master-slave mode with force feedback, as described in [8], using the hand- 

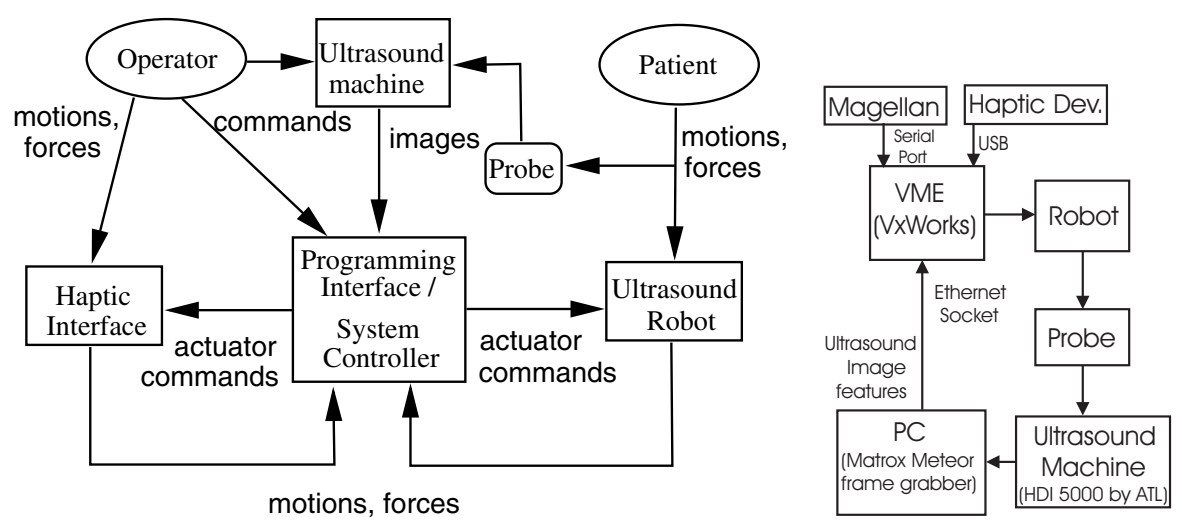

Fig. 5. Signal flow and present hardware implementation for the teleoperation system

controller described in [7]. Feasibility experiments have been carried out [8].

Shared operator/robot controller mode, in which the two modes specified above might be used along some of the degrees of freedom, but not others. For example, the probe force along its beam center axis $z_{f}$ could be controlled or kept within safe pre-specified values by the robot controller.

Shared operator/robot controller/image processor control, in which visual servoing can be used to control up to three degrees of freedom - the translation and rotation of the ultrasound transducer in the plane of the ultrasound beam, while the operator and robot controller control the other degrees of freedom. The image Jacobian obtained below demonstrates that indeed the three degrees of freedom in the ultrasound image plane are controllable, while the ability to track features in ultrasound images over tens of seconds with a modified image correlation technique has also been demonstrated and is summarized in Section 4.

\subsection{Force/Velocity Control}

Because the robot is counterbalanced and velocities are small, the robot dynamics simplify to

$$
M(\theta) \cdot \ddot{\theta}=\tau-J^{T} \cdot F
$$

where $\theta \in R^{6}$ denotes the robot joint variables, $M(\theta) \in R^{6 \times 6}$ is the joint space mass matrix, $\tau \in R^{6}$ denotes the control torques, $J \in R^{6 \times 6}$ is the Jacobian matrix, and $F \in R^{6}$ is the force/moment exerted on the patient.

The control approach is illustrated in Fig. 6. Its objective is to let a linear combination of the ultrasound probe velocity and scaled force track the joystick command (displacement), i.e.

$$
\dot{X}+K_{f} \cdot F=\text { Command, }
$$


where $\dot{X} \in R^{6}$ denotes the linear/angular velocity of the end-effector and $K_{f}$ is a force scaling matrix. When in free motion where $F=0$, the ultrasound transducer Cartesian velocity tracks the command; while in contact motion where $\dot{X}$ is very small, the contact force is controlled by the command proportionally. There is no explicit switching between the contact and free motion states.

The joint space control law in Fig. 6 is designed as where $e=\theta_{d}-\theta$,

$$
\begin{aligned}
\tau= & M(\theta) \cdot\left[\ddot{\theta}_{d}+\lambda \cdot \dot{e}\right] \\
& +K_{s} \cdot S+K_{I} \cdot S^{*} \\
\ddot{\theta}_{d}= & C \cdot\left(\dot{\theta}_{d}^{*}-\dot{\theta}_{d}\right) \\
S= & \dot{e}+\lambda \cdot(e+\zeta) \\
\dot{S}_{i}^{*}= & \begin{cases}S_{i} \underline{S}^{*} \leq S_{i}^{*} \leq \bar{S}_{i} \\
0 & \text { otherwise }\end{cases} \\
\zeta_{i}= & \begin{cases}\delta_{i}-e_{i}, & e_{i}+\xi_{i}>\delta_{i} \\
-\delta_{i}-e_{i}, & e_{i}+\xi_{i}<-\delta_{i}(8) \\
\zeta_{i} & \text { otherwise }\end{cases}
\end{aligned}
$$

the subscript $i$ denotes the $i^{\text {th }}$ entry of a vector, $\lambda>0$ is a control parameter determining the compliance of the robot arm, $\delta$ is a vector of small positive threshold elements, and $K_{s}$ and $K_{I}$ are two positive-definite gain matrices. $C$ is a filter parameter, and $S^{*}$ is an integral term with lower and upper bounds $\underline{S^{*}}$ and $\overline{S^{*}}$, respectively.

The control law (4) implements a joint-space PID controller with saturated integral terms and mass and acceleration feedforward, modified by a "reset" function $\zeta$ defined in (8) that never allows position errors to be large. Under normal operation, absolute joint position errors will be limited by $\delta_{i}$, i.e. $\left|(e+\zeta)_{i}\right| \leq \delta_{i}$. Should the patient or the ultrasound technician attempt to push the robot out of the way, $\zeta$ becomes active in such a way that the absolute position errors are still bounded by $\delta$. Should the robot return to normal operation, it will stay close to where it was released, with an error determined by the size of $\delta$. Note that the reset function does not require force sensor input. The natural low stiffness of the arm allows it to be moved away by pushing on any part of the arm linkage. The proportional and integral control terms are not allowed to increase, and therefore wind-up effects (the robot swinging when the patient's push subsides) do not exist.

The contact force $F$ is handled in two ways. First, it is treated as a disturbance in the robot dynamics and is compensated by $S^{*}$. Second, $F$ is measured by the force sensor and is used to achieve the control objective (3).

\subsection{Visual Servoing and Feature Tracking}

Visual servo-control [2] could be used to control motion in the plane of the ultrasound beam. Its feasibility can be determined by examining the ultrasound image 
Jacobian $J_{v}$, which relates differential changes in image features to differential changes in the configuration of the robot [2]. Let $p_{i}$ be a feature point in the plane of the ultrasound beam with coordinates $\left[{ }^{f} x_{i},{ }^{f} y_{i},{ }^{f} z_{i}\right]^{T}$ in the probeattached frame. Assuming an orthographic projection model with scale $a$ for the ultrasound image and that $p_{i}$ remains in the image plane, the coordinates of $p_{i}$ in the two-dimensional ultrasound image become $\left[0, u_{i}, v_{i}\right]^{T}=\left[\begin{array}{ll}0,{ }^{f} y_{i} & a^{f} z_{i}\end{array}\right]^{T}$. It can be shown that

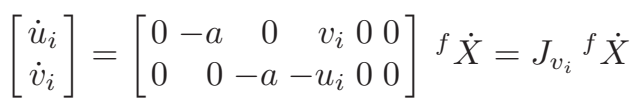

where ${ }^{f} \dot{X}$ are the translational and angular end-effector velocities in end-effector coordinates. If several points are considered in the image, similar pairs of rows will be added to (9). The rank of the resulting Jacobian is at most three. Two or more feature points non-colinear with the origin will generate a Jacobian of rank three. Thus, as expected, with non-trivial ultrasound images, it is possible to control the motion of the ultrasound transducer in its image plane.

\section{Experimental Results}

\subsection{Teleoperation Control}

The control approach proposed in Section 3.1 was implemented with the hardware described in Fig. 5, with the mass matrix identified at the nominal configuration of the robot (shown in Figure 3) by applying small joint torques. Figs. 7 to 9 demonstrate the effectiveness of the control law while the operator controls the robot in vertical motion. Scaling parameters were set such that the maximum $1.5 \mathrm{~mm}$ joystick motion corresponds to a transducer velocity of $0.18 \mathrm{~m} / \mathrm{s}$ and a force of $36 \mathrm{~N}$. Actual forces are limited to $20 \mathrm{~N}$ by the robot capability and to $10 \mathrm{~N}$ by software and hardware.

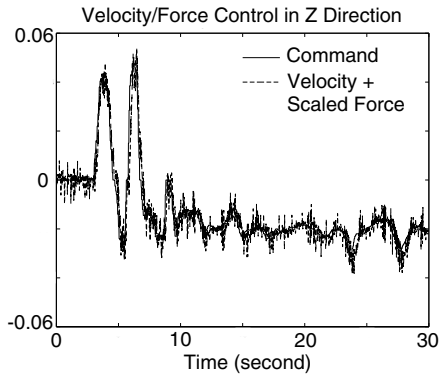

Fig. 7. Tracking of velocity plus scaled force vs command in the $z_{p}$ direction Free motion with velocity tracking is displayed until approximately $t=10 \mathrm{~s}$, when the probe contacts a person's forearm until $t=30 \mathrm{~s}$.

Fig. 7 illustrates the excellent tracking between the command and the linear combination of velocity and scaled force. Fig. 8 shows excellent velocity tracking in free motion, but not in contact motion. Fig. 9 demonstrates excellent tracking of scaled force in contact motion. It is clear that the both position and forces are followed precisely with appropriate switching between free motion and contact.

Some of the safety features built into the controller and robot design were also successfully tested. The robot could be easily moved away with a single hand, and would remain put in the position in which it was left by the operator. Turning the motor current drivers off left the robot in equilibrium. 


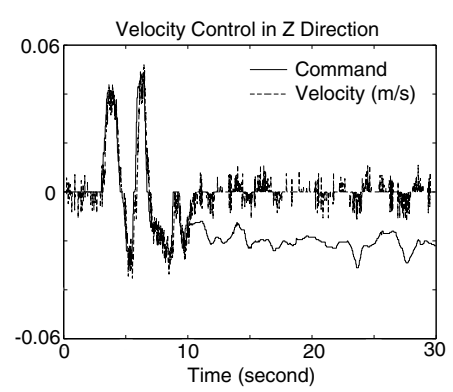

Fig. 8. Velocity tracking

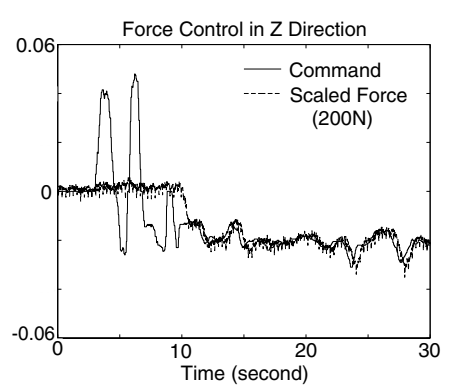

Fig. 9. Force tracking

\subsection{Feature Tracking in Ultrasound Images}

Motion tracking of a region of interest in B-mode ultrasound images was demonstrated before (see, e.g., [9]), but with different goals. Of particular interest to the problem of visual servoing and shared control is the ability to track images over a long period of time. A normalized cross-correlation technique was modified for this purpose and is presented here.

Video-images from an ultrasound examination were obtained. Typical transverse scans clearly showing the carotid artery are shown in Fig. 10. The video images were digitized and processed using a $233 \mathrm{MHz}$ Pentium II PC with a Matrox Meteor frame-grabber.

In a normalized cross-correlation technique, a sub-block of the image (in this case, a $128 \times 128$ pixel area containing the carotid artery) acquired at time $t_{i}$ is shifted in its vicinity looking for a best correlated match with a fixed sub-block of the same size in a prior frame $t_{k}$. If $k$ is fixed, the best correlation is sought relative to a fixed or reference image. Applying the cross-correlation method in this way leads to little drift, but high sensitivity to image deformation. If $i-k$ is fixed, the best correlation is sought relative to an image acquired a fixed time offset relative to the current frame. Applying the cross-correlation method in this way leads to little sensitivity to image deformation, but to significant drift, as the shift estimate is being integrated. A mixed approach was implemented that seeks the best correlation relative to multiple frames at times $t_{k}, t_{k-2}, t_{k-4}$, $\ldots, t_{k-2^{n}}$, where $n$ is fixed.

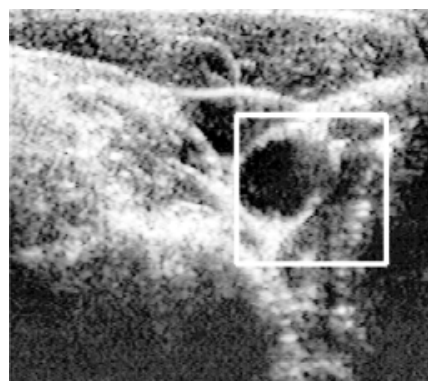

(a) Initial image.

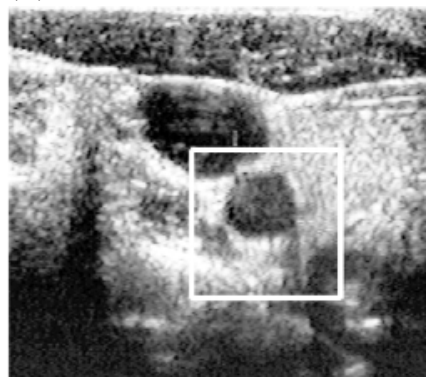

(b) Final image.

Fig. 10. Images of the carotid artery taken ten seconds apart. 
Assuming only translational displacements, image sub-blocks of $128 \times 128$ of the carotid artery could be tracked at 30 frames/second most often in the range of 5 to 10 seconds, and for as long as 30 seconds.

\section{Conclusions}

A robot-assisted system for performing ultrasound examinations was proposed in this paper with the goal of providing a more ergonomic interface for sonographers. A prototype problem, that of carotid artery examination, was considered. A novel safe robot with a large workspace was designed and built and its control in velocity/force mode was demonstrated. The feasibility of tracking ultrasound image blocks over significant time intervals was also demonstrated. In the immediate future, the system will be used to explore the use of operator-computer shared control of the ultrasound probe and ultrasound image servoing. It is hoped that other applications of the system, including the ability to acquire 3-D images and to perform precise registration, will also be developed.

\section{Acknowledgments}

Discussions with Prof. David Lowe and Dr. Paul Trepanier, help with imaging from Henry Wong, machining and robot construction by David Fletcher and Peter Vautour are gratefully acknowledged. This work is supported by the Canadian IRIS Network of Centres of Excellence project SAL.

\section{References}

1. H. E. Vanderpool, E. A. Friis, B. S. Smith, and K. L. Harms, "Prevalence of carpal tunnel syndrome and other work-related musculoskeletal problems in cardiac sonographers," Journal of Occupational Medicine, vol. 35, pp. 604-610, June 1993. 1062

2. P. I. Corke, Visual Control of Robots: High Performance Visual Servoing, John Wiley \& Sons Inc., 1996. 1063, 1068, 1069

3. J. W. Sublett, B. J. Dempsey, and A. C. Weaver. Design and implementation of a digital teleultrasound system for real-time remote diagnosis. In Proceedings of the 1995 Sympo-sium on Computer-Based Medical Systems, pages 292-298, 1995. 1063

4. J. F. Brinkley, W. E. Moritz, and D. W. Baker. Ultrasonic three-dimensioanl imaging and volume from a series of arbitrary sector scans. Ultrasound in Medicine and Biology, 4:317-327, 1978. 1063

5. R. H. Taylor, J. Funda, B. Eldridge, S. Gomory, K. Gruben, D. LaRose, M. Talamini, L. Kavoussi, and J. Anderson. A telerobotic assistant for laparoscopic surgery. IEEE Engineering in Medicine and Biology Magazine, 14(3):279-288, May/June 1995. 1065

6. Hirzinger, G., Dietrich, J., Gombert, B., Heindl, J., Landzettel, K., Schott, J., "The sensory and telerobotic aspects of the space robot experiment ROTEX," in Int. Symp. on Artificial Intel., Rob. and Aut. in Space, (Toulouse, France), Sept. 30- Oct. 2, 1992. 1066 
7. S.E. Salcudean and N.R. Parker, "6-DOF Desk-Top Voice-Coil Joystick", Symp. Haptic Interfaces for Virtual Env. and Teleop. Syst., Intl. Mech. Eng. Congr. Exp., DSC-Vol. 61, pp. 131-138, Dallas, Texas, Nov. 16-21, 1997. 1067

8. W.H. Zhu and S.E. Salcudean, "Teleoperation with Adaptive Motion/Force Control", 1999 IEEE Intl. Conf. Rob. Aut., pp. 231-237, Detroit, USA, May 1999. 1066, 1067

9. E.J. Chen, I.A. Hein, J.B. Fowles, R.S. Adler, P.L. Carson, and W.D. O'Brien Jr., "A Comparison of the Motion Tracking of 2-D Ultrasonic B-Mode Tissue Images with a Calibrated Phantom", 1991 IEEE Ultrasonic Symposium, pp. 1211-1213. 1070 